To appear in the International Journal of Computer Mathematics

Vol. 00, No. 00, Month 20XX, 1-12

\title{
The Bicube: An Interconnection of Two Hypercubes
}

\author{
Hyeong-Seok Lim ${ }^{a}$, Jung-Heum Park $^{b}$, and Hee-Chul Kim ${ }^{c *}$ \\ ${ }^{a}$ School of Electronics and Computer Engineering, Chonnam National University, Gwangju, \\ Korea; ${ }^{b}$ School of Computer Science and Information Engineering, The Catholic University of \\ Korea, Bucheon, Gyeonggi-do, Korea; ${ }^{c}$ Department of Computer Science and Engineering, \\ Hankuk University of Foreign Studies, Yongin-si, Gyeonggi-do, Korea
}

(v3.0 released February 2013)

\begin{abstract}
We consider two definitions of the even-dimensional hypercube given in the literature. The labeled graphs obtained by two definitions are not same, but one is isomorphic to the other. By interconnecting two labeled graphs in such a way that each pair of vertices with the same label are joined by an edge, we construct a vertex-symmetric graph with the diameter about half that of a comparable hypercube. We extend the result to a general scheme for interconnecting two hypercubes to produce a network topology called the bicube. We show that the bicube preserves the vertex-symmetry, bipartiteness, hamiltonian and bipancyclic properties of the hypercube, and is highly edge-symmetric.
\end{abstract}

Keywords: Interconnection networks, hypercubes, diameter, vertex-symmetry, edge-symmetry

2010 AMS Subject Classification: 05C90; 68M10; 68R10

\section{Introduction}

Many interconnection network topologies have been proposed in the literature for connecting a large number of processors efficiently, for example, $[3-7,9,12,14]$. The topology of an interconnection network can be represented as a graph in which vertices and edges correspond to nodes and communication links, respectively. In this paper, we will use standard terminology in graphs (see [2]). One popular topology is the hypercube graph which has $2^{m}$ vertices and each vertex has $m$ edges incident to it. The hypercube is scalable, symmetric and has a logarithmic diameter, simple routing and broadcasting algorithms.

The diameter is an important measure for an interconnection network, because it is concerned with the maximum routing steps in transmitting a message. The hypercube does not have the smallest diameter that is possible with the degree of a vertex it has. To achieve the lower diameter than that of hypercube, a variety of hypercube variants appeared. There have been two major approaches taken so far in designing hypercube variants. In one approach, the degree of a vertex is increased by adding extra edges to create "shortcuts" between vertices, e.g. the folded hypercube [8], the enhanced hypercube [26] whose diameters are about half that of a comparable hypercube. In the other approach, the degree of a vertex is kept the same by just rearranging the hypercube edges, and the diameter can be reduced to about half, e.g. the twisted cube [12], crossed

This research was supported by Basic Science Research Program through the National Research Foundation of Korea (NRF) funded by the Ministry of Education(NRF-2013R1A1A2012890).

${ }^{*}$ Corresponding author. Email: hckim@hufs.ac.kr 
cube [7], Möbius cube [4], Mcube [25], and multiply twisted cube [6]. Also, there were some theoretical works $[5,11,29]$ that achieve even smaller diameters than the above networks, but the connection rules and routing algorithms are more complicated.

Another important measure for an interconnection network is symmetry of the network. Informally speaking, a vertex-symmetric (resp. edge-symmetric) graph looks the same from any vertex (resp. edge). The hypercube is both vertex-symmetric and edgesymmetric graph. In a symmetric network, it is easy to design parallel and communication algorithms, since it is irrelevant where the computation and/or communication starts or in which directions it will evolve. Unfortunately, the rearrangement or "twist" of the edges in the hypercube variants lose the high degree of symmetry of the hypercube, and may deteriorate the performance of the network as pointed in $[1,25]$; asymmetry in a network affects message delays under heavy traffic, and may offset the advantage of lower diameter. It is easy to find that all the above twisted cube, crossed cube, Möbius cube, Mcube, and multiply twisted cube are not vertex-symmetric.

In this paper, we present hypercube variants using two definitions of the evendimensional hypercube given in the literature. We show that two hypercubes obtained by different definitions can be combined to produce a graph with the diameter about half that of a comparable hypercube. The combined graph preserves the nice properties of the hypercube such as vertex-symmetry, bipartiteness, hamiltonian and bipancyclic properties. Also, the graph is highly edge-symmetric. We extend the result to a general scheme for interconnecting two $m$-dimensional hypercubes of every $m \geq 2$.

The organization of this paper is as follows. In the next section, we introduce some definitions and notation. In Section 3, we consider a network topology using two definitions of hypercube. In Section 4, we will give a construction scheme for interconnecting two hypercubes. Finally, in Section 5, concluding remarks of this paper will be given.

\section{Preliminaries}

We introduce definitions, notation, and some of hypercube variants concerned with this paper.

The usual definition of hypercube is as follows: An $m$-dimensional hypercube $Q_{m}$ is an $m$-regular labeled graph on $2^{m}$ vertices. Each vertex $u$ of $Q_{m}$ is labeled by a distinct binary number $u_{m} u_{m-1} \cdots u_{1}$, and two vertices are connected by an edge if and only if their binary labels differ in exactly one bit position. An edge $(u, v)$ of $Q_{m}$ is called a dimension- $d$ edge if the labels of $u$ and $v$ differ in $d$ th bit position. The dimension $d$-edges in $Q_{m}$ form a perfect matching for each $d, 1 \leq d \leq m$. Moreover, removal of all the dimension $d$-edges in $Q_{m}$ leaves two disjoint copies of $Q_{m-1}$. Conversely, $Q_{m}$ can be constructed from two $Q_{m-1}$ 's by adding a perfect matching.

For two vertices $v$ and $w$ in a graph, the distance between $v$ and $w$ is the length of the shortest path between $v$ and $w$. The diameter of a graph $G$ is the maximum distance between any two vertices of $G$.

Many interconnection networks can be constructed by connecting two lower dimensional networks. We represent the construction as follows. We are given two graphs $G_{0}$ and $G_{1}$ with $n$ vertices each. We denote by $V_{i}$ and $E_{i}$ the vertex set and edge set of $G_{i}, i=0,1$, respectively. We let $V_{0}=\left\{v_{1}, v_{2}, \ldots, v_{n}\right\}$ and $V_{1}=\left\{w_{1}, w_{2}, \ldots, w_{n}\right\}$. With respect to a permutation $M=\left(i_{1}, i_{2}, \ldots, i_{n}\right)$ of $\{1,2, \ldots, n\}$, we can "merge" the two graphs into a graph $G_{0} \oplus_{M} G_{1}$ with $2 n$ vertices in such a way that the vertex set $V=V_{0} \cup V_{1}$ and the edge set $E=E_{0} \cup E_{1} \cup E_{2}$, where $E_{2}=\left\{\left(v_{j}, w_{i_{j}}\right): 1 \leq j \leq n\right\}$. We denote by $G_{0} \oplus G_{1}$ a graph obtained by merging $G_{0}$ and $G_{1}$ w.r.t. an arbitrary permutation $M$. Obviously, $E_{2}$ is a perfect matching in $G_{0} \oplus G_{1}$. 
Vaidya et al. [27] introduced a class of hypercube-like interconnection networks, called $H L$-graphs which can be defined by applying the $\oplus$ operation repeatedly as follows: $H L_{0}=\left\{K_{1}\right\}$; for $m \geq 1, H L_{m}=\left\{G_{0} \oplus G_{1}: G_{0}, G_{1} \in H L_{m-1}\right\}$. Then, $H L_{1}=\left\{K_{2}\right\}$; $H L_{2}=\left\{C_{4}\right\} ; H L_{3}=\left\{Q_{3}, G(8,4)\right\}$. Here, $C_{4}$ is a cycle graph with 4 vertices, $Q_{3}$ is a 3 -dimensional hypercube, and $G(8,4)$ is a recursive circulant which is isomorphic to twisted cube $T Q_{3}$ and Möbius ladder. An arbitrary graph which belongs to $H L_{m}$ is called an $m$-dimensional $H L$-graph. Note that each graph in $H L_{m}$ is $m$-regular and has $2^{m}$ vertices. Many well-known interconnection networks are members of HL-graphs including hypercube [17], twisted cube [12], twisted $m$-cube [9], crossed cube [7], Möbius cube [4], Mcube [25], multiply twisted cube [6], locally twisted cube [28], generalized twisted cube [3], and recursive circulant $G\left(2^{m}, 4\right)$ [20]. There have been many works on the fundamental properties of the above networks such as diameter, connectivity, hamiltonicity, and the embedding of other networks. Also, some literature [16, 19, 21-24] presented the generalized results on HL-graphs. Most of the interconnection networks in HL-graphs having smaller diameters than that of the hypercube are based on $G(8,4)$. That is, using $G(8,4)$ as a base case, those networks can be constructed inductively by the $\oplus$ operation specified in their definitions.

The $m$-dimensional folded hypercube, $F H_{m}$, is constructed by adding a complementary edge to $Q_{m}$ between every pair of vertices with complementary labels. Due to the complementary edge, $F H_{m}$ is an $(m+1)$-regular graph and its diameter is $\lceil m / 2\rceil$.

Two graphs $G$ and $H$ are isomorphic if there exists an isomorphism $f: V(G) \rightarrow V(H)$ such that any two vertices $u$ and $v$ of $G$ are adjacent in $G$ if and only if $f(u)$ and $f(v)$ are adjacent in $H$.

\section{Interconnection of Two Even-Dimensional Hypercubes}

In this section, we introduce an equivalent definition of hypercube in [13] that is different from the usual one given in Section 2. Then, we consider the symmetry of the graph constructed by combining two hypercubes obtained by mutually different definitions.

We can think of a graph with $2^{m}$ vertices whose vertices are labeled by $m$-bit binary numbers and two vertices are joined by an edge if their binary labels differ in exactly $k$ bit positions. It becomes a regular graph of degree $\left(\begin{array}{c}m \\ k\end{array}\right)$. Of course, when $k=1$, the graph is the very $m$-dimensional hypercube. For $k=m-1$, the graph is $m$-regular, too. Such a graph was considered in [13] as follows:

DeFinition 3.1 An m-dimensional graph $Q_{m}^{\prime}$ is an $m$-regular labeled graph on $2^{m}$ vertices. Each vertex u of $Q_{m}^{\prime}$ is labeled by a distinct binary number $u_{m} u_{m-1} \cdots u_{1}$, and two vertices are connected by an edge if and only if their binary labels differ in exactly $m-1$ bit positions.

An edge $(u, v)$ of $Q_{m}^{\prime}$ is called a dimension-d edge if the labels of $u$ and $v$ are the same in $d$ th bit position. If $m$ is an odd integer, every edge of $Q_{m}^{\prime}$ joins two vertices whose labels have the same parity. A binary label has even parity if it contains an even number of 1's and odd parity if it contains an odd number of 1's. Thus, the graph $Q_{m}^{\prime}$ for odd $m$ is disconnected. It has two connected components of the same size. If $m$ is an even integer, every edge of $Q_{m}^{\prime}$ joins two vertices whose labels are different in parity. The graph $Q_{m}^{\prime}$ is bipartite and its vertex set is partitioned into two partite sets: the set of vertices with even-parity labels and set of vertices with odd-parity labels.

Interestingly, it was shown in [13] that $Q_{m}^{\prime}$ is isomorphic to $Q_{m}$ for every even $m$ and $Q_{m}^{\prime}$ is isomorphic to two copies of $F H_{m-1}$ for every odd $m, m \geq 2$. Although the isomorphic result on $Q_{m}$ and $Q_{m}^{\prime}$ of even $m$ is already known, we give a simple and 
intuitive proof in the following for later use.

LEMMA $3.2([13]) \quad Q_{m}^{\prime}$ is isomorphic to $Q_{m}$ for any even $m \geq 2$.

Proof. Let $f: V\left(Q_{m}^{\prime}\right) \rightarrow V\left(Q_{m}\right)$ be a function such that

$$
f(x)=\left\{\begin{array}{l}
x^{\prime} \text { if the label of } x \text { has even parity } \\
x^{c} \text { if the label of } x \text { has odd parity }
\end{array}\right.
$$

where $x^{\prime}$ is the vertex with the same label as $x$, and $x^{c}$ is the vertex with a label complemented to the label of $x$. Obviously, $f$ is a bijection. We claim that $(x, y) \in E\left(Q_{m}^{\prime}\right)$ if and only if $(f(x), f(y)) \in E\left(Q_{m}\right)$. It suffices to show the 'only if' part since both graphs have the same number of edges. Let $(x, y)$ be an edge of $Q_{m}^{\prime}$ and assume that the label of $x$ has even parity and the label of $y$ has odd parity. The fact that labels of $x$ and $y$ differ in $m-1$ bit positions implies that labels of $x^{\prime}$ and $y^{c}$ differ in one bit position. Thus, $\left(x^{\prime}, y^{c}\right)$ is an edge of $Q_{m}$. This completes the proof.

The graph with $2^{m}$ vertices obtained from $Q_{m-1}$ and $Q_{m-1}^{\prime}$ by simply joining each pair of vertices of the same label via an edge, denoted by $G_{m}$, possesses interesting properties in symmetry and diameter.

DEFinition 3.3 For an odd integer $m \geq 3$, the graph $G_{m}$ is defined as follows: $V\left(G_{m}\right)=$ $V\left(Q_{m-1}\right) \cup V\left(Q_{m-1}^{\prime}\right)$ and $E\left(G_{m}\right)=E\left(Q_{m-1}\right) \cup E\left(Q_{m-1}^{\prime}\right) \cup E^{\prime}$ where $E^{\prime}=\{(u, v): u \in$ $V\left(Q_{m-1}\right), v \in V\left(Q_{m-1}^{\prime}\right)$, and the labels of $u$ and $v$ are the same $\}$.

We let dimension- $d$ edges of $G_{m}$ be the dimension- $d$ edges of $Q_{m-1}$ and $Q_{m-1}^{\prime}, 1 \leq d \leq$ $m-1$. And let dimension- $m$ edges of $G_{m}$ be the edges in $E^{\prime}$. The label of each vertex in $G_{m}$ is denoted by prefixing a bit 0 to each vertex label in $Q_{m-1}$ and prefixing a bit 1 to each vertex label in $Q_{m-1}^{\prime}$ so that each vertex of $G_{m}$ is labeled by a distinct $m$-bit binary number. The graph $G_{m}$ is bipartite and consists of two partite sets, the set of vertices with even-parity labels and the set of vertices with odd-parity labels. Further, it is equitable, that is, the two partite sets are of the same size.

We say that a graph $G$ is vertex-symmetric if any two vertices of $G$ are similar, that is, if for any $u, v$ in $V(G)$, there is an automorphism $g$ of $G$ such that $g(u)=v$. Informally speaking, every vertex of a vertex-symmetric graph looks exactly the same as every other vertex.

THEOREM $3.4 G_{m}$ is vertex-symmetric for odd $m \geq 3$.

Proof. Within this proof, we denote by $x_{m} x_{m-1} \cdots x_{1}$ the label of vertex $x$ in $G_{m}$, and the label and the vertex itself are used interchangeably. Let $u, v$ be any pair of vertices of $G_{m}$. We will show that there exists a bijection $g: V\left(G_{m}\right) \rightarrow V\left(G_{m}\right)$ such that $g(u)=v$ and for any edge $(x, y)$ of $G_{m},(g(x), g(y))$ is also an edge of $G_{m}$. Define an $m$-bit mask $M:=M_{m} M_{m-1} \cdots M_{1}=u$ XOR $v$, that is, $M=u_{m} u_{m-1} \cdots u_{1}$ XOR $v_{m} v_{m-1} \cdots v_{1}$. Here, XOR denotes the bitwise exclusive-OR operation. Notice that two labels $x$ and $y$ differ in $k$ bit positions if and only if $x$ XOR $M$ and $y$ XOR $M$ differ in $k$ bit positions. This is because $x_{i}=y_{i}$ if and only if $x_{i}$ XOR $M_{i}=y_{i}$ XOR $M_{i}$. There are two cases depending on whether $u_{m}=v_{m}$ or not.

Case 1: $u_{m}=v_{m}$.

Remember $M_{m}=0$ in this case. We define $g$ as follows:

$$
g(x)=x \text { XOR } M
$$

It is straightforward to check that $g$ is bijective and $g(u)=u$ XOR $M=$ 
$u$ XOR $(u$ XOR $v)=v$. Let $(x, y)$ be an arbitrary edge of $G_{m}$. When $(x, y)$ is an edge of subgraph $Q_{m-1}$ (resp. $Q_{m-1}^{\prime}$ ), we have $x_{m}=y_{m}=0$ (resp. $\left.x_{m}=y_{m}=1\right)$ ). Letting $g(x)=x_{m}^{\prime} x_{m-1}^{\prime} \cdots x_{1}^{\prime}$ and $g(y)=y_{m}^{\prime} y_{m-1}^{\prime} \cdots y_{1}^{\prime}$, we have $x_{m}^{\prime}=y_{m}^{\prime}=0$ (resp. $x_{m}^{\prime}=y_{m}^{\prime}=1$ ), and $x_{m-1}^{\prime} \cdots x_{1}^{\prime}$ and $y_{m-1}^{\prime} \cdots y_{1}^{\prime}$ differ in 1 (resp. $m-2$ ) bit position. Thus, $(g(x), g(y))$ is an edge of $G_{m}$. When $(x, y)$ is a dimension- $m$ edge, we have $x_{m} \neq y_{m}$ and $x_{m-1} x_{m-2} \cdots x_{1}=y_{m-1} y_{m-2} \cdots y_{1}$. Then, $g(x)$ and $g(y)$ differ in $m$ th bit position and are the same in all the other bit positions, thus $(g(x), g(y))$ is also a dimension- $m$ edge of $G_{m}$.

Case 2: $u_{m} \neq v_{m}$.

In this case, $M_{m}=1$. The function $g$ is defined as follows:

$$
g(x)=\left\{\begin{array}{l}
x_{m} x_{m-1} x_{m-2} \cdots x_{1} \text { XOR } M \\
\text { if } x_{m-1} x_{m-2} \cdots x_{1} \text { has the same parity as } u_{m-1} u_{m-2} \cdots u_{1} \\
x_{m} \frac{x_{m-1} x_{m-2} \cdots x_{1}}{\text { XOR } M \text { otherwise. }}
\end{array}\right.
$$

Here, $\overline{x_{m-1} x_{m-2} \cdots x_{1}}$ denotes the complement of $x_{m-1} x_{m-2} \cdots x_{1}$. Obviously, $g$ is bijective. It holds true that $g(u)=u$ XOR $M=u$ XOR $(u$ XOR $v)=v$. Let $(x, y)$ be an arbitrary edge of $G_{m}$. When $x_{m}=y_{m}$, we assume w.l.o.g. $x_{m-1} x_{m-2} \cdots x_{1}$ has the same parity as $u_{m-1} u_{m-2} \cdots u_{1}$. Of course, $y_{m-1} y_{m-2} \cdots y_{1}$ differs from $u_{m-1} u_{m-2} \cdots u_{1}$ in parity. If $x_{m}=y_{m}=0$ (resp. $\left.x_{m}=y_{m}=1\right)$, and $x_{m-1} x_{m-2} \cdots x_{1}$ and $y_{m-1} y_{m-2} \cdots y_{1}$ differ in 1 (resp. $m-2$ ) bit position, then $x_{m-1} x_{m-2} \cdots x_{1}$ and $\overline{y_{m-1} y_{m-2} \cdots y_{1}}$ differ in $m-2$ (resp. 1) bit positions. Then, we have $x_{m}^{\prime}=y_{m}^{\prime}=1$ (resp. $x_{m}^{\prime}=y_{m}^{\prime}=0$ ), and $x_{m-1}^{\prime} \cdots x_{1}^{\prime}$ and $y_{m-1}^{\prime} \cdots y_{1}^{\prime}$ differ in $m-2$ (resp. 1) bit positions, where $g(x)=x_{m}^{\prime} x_{m-1}^{\prime} \cdots x_{1}^{\prime}$ and $g(y)=y_{m}^{\prime} y_{m-1}^{\prime} \cdots y_{1}^{\prime}$. This implies $(g(x), g(y))$ is an edge of $G_{m}$. Finally, let $x_{m} \neq y_{m}$. Then, $(x, y)$ is a dimension- $m$ edge. We have $x_{m-1} x_{m-2} \cdots x_{1}=y_{m-1} y_{m-2} \cdots y_{1}$. Whether $x_{m-1} x_{m-2} \cdots x_{1}$ has the same parity as $u_{m-1} u_{m-2} \cdots u_{1}$ or not, we can see that $x_{m}^{\prime} \neq y_{m}^{\prime}$ and $x_{m-1}^{\prime} x_{m-2}^{\prime} \cdots x_{1}^{\prime}=y_{m-1}^{\prime} y_{m-2}^{\prime} \cdots y_{1}^{\prime}$. Thus, $(g(x), g(y))$ is an edge of $G_{m}$. This completes the proof.

Remark 1 The automorphism $g$ of $G_{m}$ given in the proof of Theorem 3.4 preserves the partition $V\left(Q_{m-1}\right)$ and $V\left(Q_{m-1}^{\prime}\right)$ of $V\left(G_{m}\right)$, that is, either $\left\{g(x): x \in V\left(Q_{m-1}\right)\right\}=$ $V\left(Q_{m-1}\right)$ and $\left\{g(y): y \in V\left(Q_{m-1}^{\prime}\right)\right\}=V\left(Q_{m-1}^{\prime}\right)$ or $\left\{g(x): x \in V\left(Q_{m-1}\right)\right\}=V\left(Q_{m-1}^{\prime}\right)$ and $\left\{g(y): y \in V\left(Q_{m-1}^{\prime}\right)\right\}=V\left(Q_{m-1}\right)$.

Associated with any automorphism, there is an induced mapping on the edges obtained by considering the image of two end-vertices of any edge. Two edges $(u, v)$ and $(x, y)$ are similar if there exists an automorphism $h$ of $G$ such that maps $(u, v)$ to $(x, y)$, i.e., $h(u)=x \& h(v)=y$ or $h(u)=y \& h(v)=x$. Notice that the 'similarity' relation on the set of edges is an equivalence relation. The graph $G_{m}$ keeps high degree of edge symmetry, precisely speaking, its edge set can be partitioned into two equivalence classes as follows.

Theorem 3.5 Any two dimension- $m$ edges in $G_{m}$ with odd $m \geq 3$ are similar, and any two edges which are not dimension-m edges are also similar.

Proof. We first show that two dimension- $m$ edges $(u, v)$ and $(a, b)$ are similar. Assume $u_{m}=0$ and $a_{m}=0$. Thus, $v_{m}=1$ and $b_{m}=1$. Letting an $m$-bit mask $M=u$ XOR $a$, we define automorphism $h$ of $G_{m}$ as follows:

$$
h(x)=x \text { XOR } M .
$$

Then, $h(u)=u$ XOR $M=u$ XOR $(u$ XOR $a)=a$. Furthermore, since $M=u$ XOR $a=$ $v$ XOR $b$, we have $h(v)=v$ XOR $M=v$ XOR $(v$ XOR $b)=b$. Here, $M_{m}=0$. In exactly the 
same way to Case 1 of the proof of Theorem 3.4, we can show that if $(x, y)$ is an edge of $G_{m}$, then $(h(x), h(y))$ is also an edge of $G_{m}$.

Now, we are to show two edges $(u, v)$ and $(a, b)$ with $u_{m}=v_{m}=a_{m}=b_{m}=0$ are similar. Here, $(u, v)$ and $(a, b)$ are edges of subgraph $Q_{m-1}$. We assume $u_{i}=0$ and $v_{i}=1$ for some $i<m$, and assume $a_{j}=0$ and $b_{j}=1$ for some $j<m$. Let $x^{i, j}$ be the $m$-bit binary number obtained from $x$ by swapping $i$ th bit and $j$ th bit of $x$, possibly $i=j$ where $1 \leq i, j<m$. That is, $x^{i, j}=x_{m} \cdots x_{i+1} x_{j} x_{i-1} \cdots x_{j+1} x_{i} x_{j-1} \cdots x_{1}$. Define $m$-bit mask $M=u^{i, j}$ XOR $a$. Then, we have $M=u^{i, j}$ XOR $a$

$=u_{m} \cdots u_{i+1} u_{j} u_{i-1} \cdots u_{j+1} u_{i} u_{j-1} \cdots u_{1}$ XOR $a_{m} \cdots a_{i+1} a_{i} a_{i-1} \cdots a_{j+1} a_{j} a_{j-1} \cdots a_{1}$

$=u_{m} \cdots u_{i+1} u_{j} u_{i-1} \cdots u_{j+1} \overline{u_{i}} u_{j-1} \cdots u_{1}$ XOR $a_{m} \cdots a_{i+1} a_{i} a_{i-1} \cdots a_{j+1} \overline{a_{j}} a_{j-1} \cdots a_{1}$

$=v^{i, j}$ XOR $b$. An automorphism $h$ of $G_{m}$ is defined as follows:

$$
h(x)=x^{i, j} \text { XOR } M .
$$

Then, we have $h(u)=u^{i, j}$ XOR $M=u^{i, j}$ XOR $\left(u^{i, j}\right.$ XOR $\left.a\right)=a$ and $h(v)=v^{i, j}$ XOR $M$ $=v^{i, j}$ XOR $\left(v^{i, j}\right.$ XOR $\left.b\right)=b$. Observe that $x$ and $y$ differ in $k$ bit positions if and only if $x^{i, j}$ and $y^{i, j}$ differ in $k$ bit positions. Remembering the observation and $M_{m}=0$, we can show in a very similar way to Case 1 of proof of Theorem 3.4 that for any edge $(x, y)$ of $G_{m},(h(x), h(y))$ is also an edge of $G_{m}$.

Recall that the 'similarity' relation on the set of edges is an equivalence relation. It suffices to show that for any edge $(x, y)$ with $x_{m}=y_{m}=1$, there is an edge of $Q_{m-1}$ which is similar to $(x, y)$. Let $u$ be an arbitrary vertex with $u_{m}=0$. By Theorem 3.4, there exists an automorphism $g$ of $G_{m}$ such that $g(x)=u$. Let $v=g(y)$. Since $g$ is an automorphism, $(u, v)$ is an edge of $G_{m}$. Furthermore, by Remark $1, v$ is also a vertex with $v_{m}=0$. Thus, $(x, y)$ is similar to the edge $(u, v)$ of $Q_{m-1}$. This completes the proof.

Remark 2 A graph is edge-symmetric if every pair of edges are similar. $G_{m}$ is not edgesymmetric for all odd $m \geq 5$. There is a cycle of length 4 passing through an arbitrary dimension- $p$ edge, $p<m$. We claim that there is no cycle of length 4 passing through a dimension- $m$ edge $(u, v)$. We assume $u \in V\left(Q_{m-1}\right)$ and $v \in V\left(Q_{m-1}^{\prime}\right)$. The labels of $u$ and any vertex $u^{\prime} \in V\left(Q_{m-1}\right)$ adjacent to $u$ differ in 1 bit position, and the labels of $v$ and any vertex $v^{\prime} \in V\left(Q_{m-1}^{\prime}\right)$ adjacent $v$ differ in $(m-1)-1$ bit positions. Due to $m-2 \neq 1, u^{\prime}$ is not adjacent to $v^{\prime}$ and thus there is no such cycle.

Among the diameters of $m$-dimensional HL-graphs, the diameter of $Q_{m}$, that is $m$, is the largest. But, to our surprise, the diameter of $G_{m}$ is reduced to $(m+1) / 2$ which is about half that of the component graph $Q_{m-1}\left(\right.$ or $Q_{m-1}^{\prime}$ ) for all odd $m \geq 7$. The diameter and other nice properties of $G_{m}$ will be dealt with the generalized topology in the following section.

\section{Bicubes}

The result in Section 3 suggests that two hypercubes can be combined to produce an interesting network topology. In this section, we present a scheme for interconnecting two (not necessarily even-dimensional) hypercubes and the properties of the resulting topology, called bicube.

We are to utilize the structure $Q_{m-1} \oplus Q_{m-1}^{\prime}$ considered in Section 3. For convenience of description, we introduce some notation. Let $l(u)$ be the binary label of a hypercube vertex $u$ and $c(u)$ be the complement of $l(u)$. We will let $p()$ be the parity function that maps binary labels of the hypercube vertices to 0 or 1 . For a vertex $u$ in $Q_{m}$ with even $m, p(u)$ is defined to be 0 if the number of 1 's in $l(u)$ is even, and 1 otherwise. We call 
two vertices $u$ and $v$ are $l p$-related if either $l(u)=l(v) \& p(u)=p(v)=0$ or $l(u)=c(v)$ $\& p(u)=p(v)=1$.

Interconnecting Scheme for Bicube $B Q_{m}$

$/ *$ two $(m-1)$-dimensional hypercubes are interconnected to produce $B Q_{m}, m \geq 3 * /$

(1) Make two copies of $Q_{m-1}$, and let the copies be $Q_{m-1}^{0}$ and $Q_{m-1}^{1}$.

Case 1: $m$ odd.

Connect each lp-related pair $u \in V\left(Q_{m-1}^{0}\right)$ and $v \in V\left(Q_{m-1}^{1}\right)$ by an edge. Case 2: $m$ even.

(a) Partition $V\left(Q_{m-1}^{i}\right)$ into $V\left(Q_{m-2}^{i 0}\right)$ and $V\left(Q_{m-2}^{i 1}\right)$; vertex $v \in V\left(Q_{m-1}^{i}\right)$ belongs to $\mathrm{V}\left(Q_{m-2}^{i j}\right)$ where $j$ is the first bit of $l(v), i, j \in\{0,1\}$. Hereafter, exclude the first bit of every vertex label within this case.

(b) Connect each lp-related pair $x \in V\left(Q_{m-2}^{00}\right)$ and $y \in V\left(Q_{m-2}^{10}\right)$ by an edge.

(c) Connect each lp-related pair $w \in V\left(Q_{m-2}^{01}\right)$ and $z \in V\left(Q_{m-2}^{11}\right)$ by an edge.

(2) Prefix a bit 0 to each vertex label in $Q_{m-1}^{0}$, and prefix a bit 1 to each vertex label in $Q_{m-1}^{1}$ to produce $B Q_{m}$.

We let the dimension $d$-edges of the $B Q_{m}$ be the dimension $d$-edges in two $Q_{m-1}$ 's, $1 \leq d \leq m-1$, and let the dimension $m$-edge of the $B Q_{m}$ be the edges connected between two $Q_{m-1}$ 's. In Fig. $1, B Q_{3}, B Q_{4}$, and $B Q_{5}$ are shown. As shown in Fig. 1, $B Q_{3}$ and $B Q_{4}$ are isomorphic to $Q_{3}$ and $Q_{4}$, respectively, but $B Q_{5}$ is not isomorphic to $Q_{5}$. In fact, $B Q_{m}$ is not isomorphic to $Q_{m}$ for every $m \geq 5$, which will be clear later in Theorem 4.8.

LemMa $4.1 \quad B Q_{m}$ is isomorphic to $G_{m}$ for odd $m \geq 3$.

Proof. Similar to the proof of Lemma 3.2, if we complement all the vertex labels having odd parity (excluding the first bit) in the subgraph $Q_{m-1}^{1}$ of $B Q_{m}$, we can observe the labeled graph is same as $G_{m}$.

We note that $B Q_{m}$ with even $m$ is isomorphic to $B Q_{m-1} \times K_{2}$. In the scheme, each of $Q_{m-2}^{00} \oplus Q_{m-2}^{10}$ and $Q_{m-2}^{01} \oplus Q_{m-2}^{11}$ is interconnected to be isomorphic to $B Q_{m-1}$. So, $B Q_{m}$ with even $m$ is of structure $B Q_{m-1} \oplus_{I} B Q_{m-1}$ where $I$ is an identity permutation. Thus we have:

Lemma $4.2 B Q_{m}$ with even $m \geq 4$ is isomorphic to $B Q_{m-1} \times K_{2}$.

We already know $G_{m}$ of odd $m$ is bipartite. By lemma 4.1 and Lemma 4.2 we have the following.

Lemma $4.3 \quad B Q_{m}$ is bipartite for $m \geq 3$.

In the next, we will discuss the symmetry and diameter of $B Q_{m}$.

THEOREM $4.4 \quad B Q_{m}$ is vertex-symmetric for $m \geq 3$.

Proof. By Theorem 3.4 and Lemma 4.1, $B Q_{m}$ with odd $m$ is vertex-symmetric. $B Q_{m}$ with even $m$ is isomorphic to $B Q_{m-1} \times K_{2}$, which is vertex-symmetric obviously.

THEOREM 4.5 (a) In $B Q_{m}$ with odd $m \geq 3$, any two dimension-m edges are similar, and any two edges which are not dimension-m edges are also similar.

(b) In $B Q_{m}$ with even $m \geq 4$, any two dimension-m edges are similar, any two dimension- $(m-1)$ edges are similar, and any two edges which are neither dimension-m edges nor dimension- $(m-1)$ edges are also similar. 


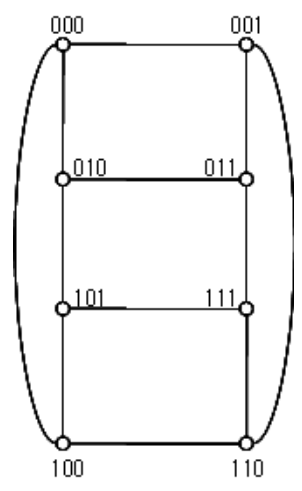

(a) $B Q_{3}$

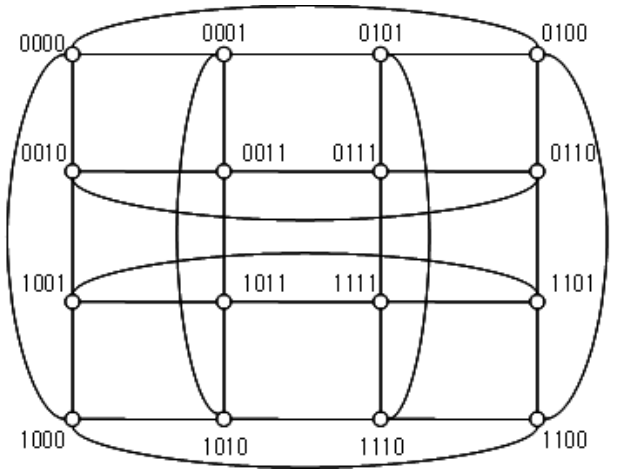

(b) $B Q_{4}$

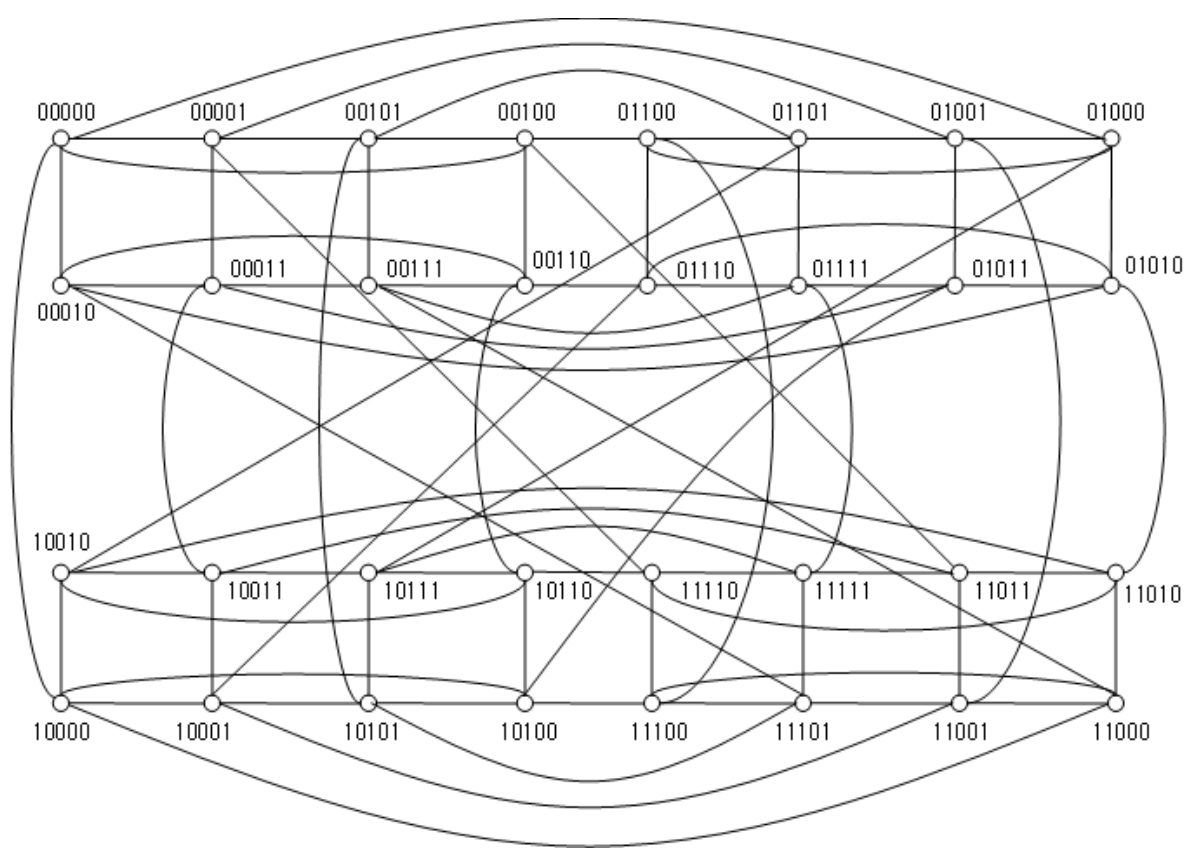

(c) $B Q_{5}$

Figure 1. Illustration of the interconnecting scheme for $B Q_{m}$

Proof. Due to Theorem 3.5 and Lemma 4.1, the statement (a) holds true. To prove (b), we let $m$ be even and denote by $B Q_{m-1}^{0}$ (resp. $B Q_{m-1}^{1}$ ) the subgraph of $B Q_{m}$ induced by vertices in $Q_{m-2}^{00}$ or $Q_{m-2}^{10}$ (resp. $Q_{m-2}^{01}$ or $Q_{m-2}^{11}$ ). Since $B Q_{m}$ is isomorphic to $B Q_{m-1} \times K_{2}$, for each edge in $B Q_{m-1}^{1}$ there exists an edge in $B Q_{m-1}^{0}$ similar to the edge, and vice versa. Recall that the 'similarity' relation on the set of edges is an equivalence relation.

We first show that any two dimension- $m$ edges are similar. Two dimension- $(m-1)$ edges in $B Q_{m-1}^{0}$ are similar within $B Q_{m-1}^{0}$ by Theorem 3.5. It is not difficult to see that they are also similar in $B Q_{m}$. Moreover, for any dimension- $(m-1)$ edge in $B Q_{m-1}^{1}$, there is a dimension- $(m-1)$ edge in $B Q_{m-1}^{0}$ which is similar to the edge. Thus, dimension$(m-1)$ edges in $B Q_{m-1}^{0}$ or $B Q_{m-1}^{1}$, which comprise dimension- $m$ edges in $B Q_{m}$, are similar each other. In the same way, we can show that any two edges which are neither dimension- $m$ edges nor dimension- $(m-1)$ edges are similar. The proof is omitted here.

It remains to show that any two dimension- $(m-1)$ edges $(u, v)$ and $(x, y)$ are similar. We assume that $u$ and $x$ are contained in $B Q_{m-1}^{0}$, and $v$ and $y$ are contained in $B Q_{m-1}^{1}$. 
Since $B Q_{m-1}$ is vertex-symmetric, there is an automorphism $g_{0}$ of $B Q_{m-1}^{0}$ mapping $u$ to $x$. Furthermore, there exists an automorphism $g_{1}$ of $B Q_{m-1}^{1}$ such that $g_{1}(w)=z$ if and only if $g_{0}\left(w^{\prime}\right)=z^{\prime}$, where $w^{\prime}$ and $z^{\prime}$ are the vertices in $B Q_{m-1}^{0}$ adjacent to $w$ and $z$, respectively. Thus, an automorphism $g$ of $B Q_{m}$ can be defined such that $g(w)=g_{0}(w)$ for $w$ in $B Q_{m-1}^{0}$ and $g(w)=g_{1}(w)$ for $w$ in $B Q_{m-1}^{1}$. Then, we have $g(u)=x$ and $g(v)=y$. Therefore, $(u, v)$ and $(x, y)$ are similar in $B Q_{m}$. This completes the proof.

Remark 3 In $B Q_{m}$ with even $m \geq 6$, a dimension- $(m-1)$ edge is not similar to any dimension- $k$ edge with $k<m-1$. The number of length 4 cycles passing through a dimension- $(m-1)$ edge is $m-1$ while that number for a dimension- $k$ edge is $m-2$ for any $k<m-1$.

LEMMA 4.6 $B Q_{m}$ of odd $m \geq 3$ is a spanning subgraph of $m$-dimensional folded hypercube, $F H_{m}$.

Proof. In $F H_{m}$, there are two sets of edges $E_{1}$ and $E_{2}$ between two copies of $Q_{m-1}$ where $E_{1}$ is a set of edges joining each pair of vertices of the same label and $E_{2}$ is a set of complementary edges. In $B Q_{m}$ of odd $m$, any edge between two $Q_{m-1}$ 's either joins two vertices of the same label, or joins two vertices with complementary labels. So, the lemma holds.

Now we consider the diameter of $B Q_{m}$. Let $0^{k}$ represent a series of $k 0$ 's. In the next, the label of a vertex and the vertex itself are used interchangeably. By virtue of the vertex-symmetry of $B Q_{m}$, it suffices to consider the distance between $0^{m}$ and other vertices. For two vertices $v$ and $w$ in $B Q_{m}$, the distance between $v$ and $w$ is denoted by $d(v, w)$, and the Hamming distance between $v$ and $w$ is denoted by $d_{H}(v, w)$.

Lemma 4.7 For $v=0^{m}$ and $w$ with $d_{H}(v, w)=k$ in $B Q_{m}$ of odd $m \geq 3$, the following distance expressions hold:

(a) For $k=m, d(v, w)=3$.

(b) For $k=m-1$ and $w \neq 01^{m-1}, d(v, w)=2$.

(c) For $k=m-1$ and $w=01^{m-1}, d(v, w)=\min \{k, 4\}$.

(d) For $k \leq m-2, d(v, w)=\min \{k, m-k+1\}$.

Proof. Proof for (a). The label of $w$ is $1^{m}$. The path $\left(0^{m}, 0^{m-1} 1,1^{m-1} 0,1^{m}\right)$ of length 3 is the shortest path between $v$ and $w$.

Proof for (b). When $k=m-1$ and $w \neq 01^{m-1}$, then $w$ is a vertex of $Q_{m-1}^{1}$. Therefore, the label of $w$ is $1^{p} 01^{q}$ for some $p, q$ such that $p \geq 1$ and $p+q=m-1$. The path $\left(0^{m}, 0^{p} 10^{q}, 1^{p} 01^{q}\right)$ of length 2 is the shortest path between $v$ and $w$.

Proof for (c). Since $d_{H}(v, w)=k$, it is obvious that there is a path of length $k$ between $v$ and $w$. The distance between $v$ and $w$ is $k$ when $k \leq 4$. There is a path $\left(0^{m}, 0^{m-1} 1,1^{m-1} 0,1^{m}, 01^{m-1}\right)$ of length 4 , which is the shortest path between $v$ and $w$ when $k \geq 5$.

Proof for (d). If $m=3$, then $k \leq 1$; if $m=5$, then $k \leq 3$. Thus, if $m=3$ or $m=5$, it is easy to see that $d(v, w)=k$ and $k \leq m-k+1$. We consider $m$ with $m \geq 7$. For each $i$ with $0 \leq i \leq m-1$, let $S_{i}^{0}=\left\{u \in V\left(\bar{Q}_{m-1}^{0}\right): d_{H}\left(0^{m}, u\right)=i\right\}$, and let $S_{i}^{1}=\left\{u \in V\left(Q_{m-1}^{1}\right)\right.$ $\left.: \bar{d}_{H}\left(10^{m-1}, u\right)=i\right\}$. Let $V_{i}(v)=\{u: d(v, u)=i\}$. That is, $V_{i}(v)$ is the set of vertices which are at distance $i$ from $v$. Note that for each $a \in S_{i}^{0}$ (resp. $S_{i}^{1}$ ), if $i$ is even there is an edge $(a, b)$ such that $b$ is in $S_{i}^{1}$ (resp. $S_{i}^{0}$ ); otherwise there is an edge $(a, b)$ such that $b$ is in $S_{m-i-1}^{1}\left(\right.$ resp. $\left.S_{m-i-1}^{0}\right)$. In $Q_{m-1}^{0}$ (resp. $Q_{m-1}^{1}$ ), the set of vertices adjacent to vertices in $S_{i}^{0}\left(\right.$ resp. $\left.S_{i}^{1}\right)$ is $S_{i-1}^{0} \cup S_{i+1}^{0}$ (resp. $S_{i-1}^{1} \cup S_{i+1}^{1}$ ) for each $i$ with $1 \leq i \leq m-2$.

For $1 \leq i \leq 4, V_{i}(v)$ is obtained as follows:

$V_{1}(v)=S_{1}^{0} \cup S_{0}^{1}$, 


$$
\begin{aligned}
& V_{2}(v)=S_{2}^{0} \cup S_{1}^{1} \cup S_{m-2}^{1}, \\
& V_{3}(v)=S_{3}^{0} \cup S_{m-2}^{0} \cup S_{2}^{1} \cup S_{m-3}^{1} \cup S_{m-1}^{1}, \text { and } \\
& V_{4}(v)=S_{4}^{0} \cup S_{m-3}^{0} \cup S_{m-1}^{0} \cup S_{3}^{1} \cup S_{m-4}^{1} .
\end{aligned}
$$

We first consider the case of $m=7$. In this case, we observe that $V_{1}(v), V_{2}(v), V_{3}(v)$, and $V_{4}(v)$ are mutually disjoint, and $\{v\} \cup V_{1}(v) \cup V_{2}(v) \cup V_{3}(v) \cup V_{4}(v)$ is equal to the set of vertices in $B Q_{m}$. From the condition of $k \leq m-2$, we have $k \leq 5$. Since $d_{H}(v, w)=k$, $w$ is in $S_{k}^{0}$ or $S_{k-1}^{1}$. If $k \leq 4, w$ is in $V_{k}(v)$ from the fact that each of $S_{k}^{0}$ and $S_{k-1}^{1}$ is a subset of $V_{k}(v)$. Therefore we have $d(v, w)=k$. Note that $m-k+1=8-k \geq k$. If $k=5$, then $w$ is in $S_{5}^{0}\left(=S_{m-2}^{0}\right)$ or $S_{4}^{1}\left(=S_{m-3}^{1}\right)$. Since each of $S_{5}^{0}$ and $S_{4}^{1}$ is a subset of $V_{3}(v)$, we have $d(v, w)=3$, which is equal to $m-k+1$.

We next consider the case of $m \geq 9$. In this case, we claim that for each $n$ with $5 \leq n \leq(m+1) / 2, V_{n}(v)=S_{n}^{0} \cup S_{m-n+1}^{0} \cup S_{n-1}^{1} \cup S_{m-n}^{1}$. We prove the claim by induction on $n$. If $n=5$, it is easy to see that the claim holds. Assuming that for each $n$ with $5 \leq n<p \leq(m+1) / 2$ the claim holds, we will show that the claim holds for $n=p$. Let $T=\{u: d(v, u) \geq p\}$. Then from the assumption, $T=\bigcup_{i=p}^{m-p+1} S_{i}^{0} \cup \bigcup_{i=p-1}^{m-p} S_{i}^{1}$. Since $V_{p}(v)=\left\{u \in T: u\right.$ is adjacent to a vertex in $\left.V_{p-1}(v)\right\}$ and $V_{p-1}(v)=S_{p-1}^{0} \cup S_{m-p+2}^{0} \cup$ $S_{p-2}^{1} \cup S_{m-p+1}^{1}$, we have $V_{p}(v)=S_{p}^{0} \cup S_{m-p+1}^{0} \cup S_{p-1}^{1} \cup S_{m-p}^{1}$. Thus, the claim is proved.

By using the claim, we will show $d(v, w)=\min \{k, m-k+1\}$. Note that $w \notin S_{m-1}^{0} \cup$ $S_{m-1}^{1} \cup S_{m-2}^{1}$ since $k \leq m-2$. If $k \leq 4$, as in the case of $m=7$, it can be shown that $d(v, w)=k$, which is less than or equal to $m-k+1$. From now on, it is assumed that $k \geq 5$. If $w$ is a vertex of $Q_{m-1}^{0}$, then $w$ is in $S_{k}^{0}$. From the claim, if $k \leq m-k+1$ (that is, $k \leq(m+1) / 2), w$ is in $V_{k}(v)$ since $S_{k}^{0}$ is a subset of $V_{k}(v)$, which means $d(v, w)=k$; otherwise, $S_{k}^{0}$ is a subset of $V_{p}(v)$ such that $m-p+1=k$, which means $d(v, w)=p=m-k+1$. If $w$ is a vertex of $Q_{m-1}^{1}, w$ is in $S_{k-1}^{1}$. From the claim, if $k-1 \leq m-k$ (that is, $k \leq(m+1) / 2), d(v, w)=k$ since $S_{k-1}^{1}$ is a subset of $V_{k}(v)$; otherwise, $S_{k-1}^{1}$ is a subset of $V_{p}(v)$ such that $m-p=k-1$, which implies $d(v, w)=p=m-k+1$. The proof is completed.

THEOREM 4.8 The diameter of $B Q_{m}$ is $\lceil(m+1) / 2\rceil$ for $m \geq 7$. The diameters of $B Q_{3}$, $B Q_{4}, B Q_{5}$ and $B Q_{6}$ are 3, 4, 4 and 5, respectively.

Proof. $B Q_{3}$ and $B Q_{4}$ are isomorphic to $Q_{3}$ and $Q_{4}$, respectively. And, it is easy to check the diameter of $B Q_{5}$ is 4 . For $B Q_{m}$ of $m \geq 7$ and odd, the maximum value in distance expression $\min \{k, m-k+1\}$ in Lemma 4.7 is obtained when $k=(m+1) / 2$. For $B Q_{m}$ of $m \geq 6$ and even, the diameter of $B Q_{m}$ is one more than the diameter of $B Q_{m-1}$ from Lemma 4.2. Together with the above facts, the theorem holds.

We note that the diameter of $B Q_{m}$ is the same as that of $F H_{m}$ for odd $m \geq 7$, even though $B Q_{m}$ is a spanning subgraph of $F H_{m}$ and the degree of $B Q_{m}$ is one less than that of $\mathrm{FH}_{m}$.

Next, we will consider hamiltonicity and pancyclicity of the $B Q_{m}$. Linear arrays and rings are two of the most important computational structures in interconnection networks. The embedding of linear arrays and rings into interconnection network has been studied in terms of paths and cycles in graphs. A graph is called hamiltonian if it has a cycle which contains all the vertices. An $s$-t hamiltonian path is a path which contains all the vertices joining a pair of vertices $s$ and $t$. If a graph has an $s$ - $t$ hamiltonian path for all pairs of vertices $s$ and $t$, the graph is called hamiltonian-connected. A bipartite graph is called hamiltonian-laceable if there is an $s$ - $t$ hamiltonian path for all pairs of vertices $s$ and $t$, where $s$ belongs to one set of the bipartition, and $t$ belongs to the other. A graph $G$ is called pancyclic if it contains a cycle of length $l$ for every $l$ such that $3 \leq l \leq|V(G)|$. And a graph $G$ is called bipancyclic if it contains a cycle of length $l$ for every even $l$, 
$4 \leq l \leq|V(G)|$. Necessarily, a hamiltonian-connected graph and pancyclic graph is not bipartite. The hamiltonicity and pancyclicity of HL-graphs were considered in [19] as follows. For more discussion on hamiltonicity and its generalization, refer to $[15,18]$.

Theorem 4.9 ([19]) Every m-dimensional bipartite HL-graph is hamiltonian-laceable, and bipancyclic for $m \geq 2$.

It is obvious that $B Q_{m}$ is a bipartite HL-graph, so the lemma follows:

LEMmA 4.10 $B Q_{m}$ is hamiltonian-laceable and bipancyclic for $m \geq 3$.

\section{Concluding remarks}

In this paper, we considered two definitions of hypercube and their application to interconnecting hypercubes. It was shown that we can obtain a graph with diameter about half that of a comparable hypercube by just interconnecting two hypercubes. Also, the graph preserves desirable properties of the hypercube. Based on the above observation, we presented an interconnection network topology called the bicube. The diameter, vertexsymmetry, edge-symmetry, hamiltonicity, and bipancyclicity of the bicube are investigated. In further research of the bicube, we expect other nice properties can be found and many algorithms developed for hypercubes can be easily adapted to bicubes.

\section{References}

[1] S. Abraham and K. Padmanabhan, The twisted cube topology for multiprocessors: a study in network asymmetry, J. Parallel Distrib. Comput. 13 (1991), pp. 104-110.

[2] J.A. Bondy and U.S.R. Murty, Graph Theory with Applications, 5th printing, American Elsevier Publishing Co., Inc., 1976.

[3] F.B. Chedid, On the generalized twisted cube, Inform. Proc. Lett. 55 (1995), pp. 49-52.

[4] P. Cull and S. Larson, The Möbius cubes, IEEE Trans. Computers 44(5) (1995), pp. 647-659.

[5] P. Cull and S. Larson, Smaller diameters in hypercube-variant networks, Telecommunication Systems 10 (1998), pp. $175-184$.

[6] K. Efe, A variation on the hypercube with lower diameter, IEEE Trans. on Computers 40(11) (1991), pp. $1312-1316$.

[7] K. Efe, The crossed cube architecture for parallel computation, IEEE Trans. on Parallel and Distributed Systems 3(5) (1992), pp. 513-524.

[8] A. El-Amawy and S. Latifi, Properties and performance of folded hypercubes, IEEE Trans. on Parallel and Distributed Systems 2(1) (1991), pp. 31-42.

[9] A.-H. Esfahanian, L.M. Ni, and B.E. Sagan, The twisted $n$-cube with application to multiprocessing, IEEE Trans. Computers 40(1) (1991), pp. 88-93.

[10] J. Fang, The bipanconnectivity and m-panconnectivity of the folded hypercube, Theoretical Computer Science 385 (2007), pp. 286-300.

[11] C.M. Hartman, Extremal problems in graph theory, Ph.D. Thesis, Dept. of Mathematics, Univ. of Illinois at Urbana-Champaign, 1997.

[12] P.A.J. Hilbers, M.R.J. Koopman, and J.L.A. van de Snepscheut, The Twisted Cube, in J. Bakker, A. Nijman, P. Treleaven, eds., PARLE: Parallel Architectures and Languages Europe, Vol. I: Parallel Architectures, Springer (1987), pp. 152-159.

[13] C.-T. Ho, An observation on the bisectional interconnection networks, IEEE Trans. Computers 41(7) (1992), pp. 873-877.

[14] S.-Y. Hsieh and T.-T. Hsiao, The k-degree Cayley graph and its topological properties, Networks 47(1) (2006), pp. 26-36.

[15] S. Jo, J.-H. Park, and K.Y. Chwa, Paired 2-disjoint path covers and strongly hamiltonian laceability of bipartite hypercube-like graphs, Information Sciences 242 (2013), pp. 102-112.

[16] J.-H. Lee and J.-H. Park, General-demand disjoint path covers in a graph with faulty elements, International Journal of Computer Mathematics 89(5) (2012), pp. 606-617. 
[17] F.T. Leighton, Introduction to Parallel Algorithms and Architectures: Arrays, Trees, Hypercubes, Morgan Kaufman, California, 1992.

[18] C.-K. Lin, Y.-H. Teng, J.M. Tan, L.-H. Hsu, and D. Marusic, The spanning laceability on the faulty bipartite hypercube-like graphs, Applied Mathematics and Computation 219(15) (2013), pp. 80958103.

[19] C.-D. Park and K.Y. Chwa, Hamiltonian properties on the class of hypercube-like networks, Inform. Proc. Lett. 91 (2004), pp. 11-17.

[20] J.-H. Park and K.Y. Chwa, Recursive circulants and their embeddings among hypercubes, Theoretical Computer Science 244 (2000), pp. 35-62.

[21] J.-H. Park, H.-C. Kim, and H.-S. Lim, Fault-hamiltonicity of hypercube-like interconnection networks, in 19th IEEE International Parallel and Distributed Processing Symposium, Denver, USA, 4-8 Apr. 2005.

[22] J.-H. Park, H.-C. Kim, and H.-S. Lim, Many-to-many disjoint path covers in hypercube-like interconnection networks with faulty elements, IEEE Trans. on Parallel and Distributed Systems 17(3) (2006), pp. 227-240.

[23] J.-H. Park, H.-C. Kim, and H.-S. Lim, Many-to-many disjoint path covers in hypercube-like interconnection networks, IEEE Trans. on Computers 58(4) (2009), pp. 528-540.

[24] J.-H. Park, H.-S. Lim, and H.-C. Kim, Panconnectivity and pancyclicity of hypercube-like interconnection networks with faulty elements, Theoretical Computer Science 377(1-3) (2007), pp. 170-180.

[25] N.K. Singhvi and K. Ghose, The Mcube: a symmetrical cube based network with twisted links, in 9th IEEE International Parallel Processing Symposium Santa Barbara, USA, 25-28 Apr. 1995, pp. $11-16$.

[26] N. Tzeng and S. Wei, Enhanced hypercubes, IEEE Trans. Computers 40(3) (1991), pp. 284-294.

[27] A.S. Vaidya, P.S.N. Rao, and S.R. Shankar, A class of hypercube-like networks, in 5th IEEE Symposium on Parallel and Distributed Processing, Dallas, USA, 1-4 Dec. 1993, pp. 800-803.

[28] X. Yang, D. J. Evans, and G. M. Megson, The locally twisted cubes, International Journal of Computer Mathematics 82(4) (2005), pp. 402-413.

[29] W. Zhou, J. Fan, X. Jia, and S. Zhang, The Spined cube: A new hypercube variant with smaller diameter, Inform. Proc. Lett. 111 (2011), pp. 561-567. 